\title{
Acute clinical care and care coordination for traumatic brain injury within Department of Defense
}

\author{
Michael S. Jaffee, MD; ${ }^{1}$ Kathy M. Helmick, CRNP; ${ }^{1}$ Philip D. Girard, MS; ${ }^{1-2 *}$ Kim S. Meyer, APRN; ${ }^{1}$ \\ Kathy Dinegar, LICSW; ${ }^{3}$ Karyn George, MS, CRC ${ }^{1}$ \\ ${ }^{1}$ Defense and Veterans Brain Injury Center, Walter Reed Army Medical Center (WRAMC), Washington, DC; \\ ${ }^{2}$ Manchester Department of Veterans Affairs Medical Center, Manchester, NH; ${ }^{3}$ WRAMC, Washington, DC
}

\begin{abstract}
The nature of current combat situations that U.S. military forces encounter and the use of unconventional weaponry have dramatically increased service personnel's risks of sustaining a traumatic brain injury (TBI). Although the true incidence and prevalence of combat-related TBI are unknown, service personnel returning from deployment have reported rates of concussion between $10 \%$ and $20 \%$. The Department of Defense has recently released statistics on TBI dating back to before the wars in Iraq and Afghanistan to better elucidate the impact and burden of TBI on America's warriors and veterans. Patients with severe TBI move through a well-established trauma system of care, beginning with triage of initial injury by first-responders in the war zone to acute care to rehabilitation and then returning home and to the community. Mild and moderate TBIs may pose different clinical challenges, especially when initially undetected or if treatment is delayed because more serious injuries are present. To ensure identification and prompt treatment of mild and moderate TBI, the U.S. Congress has mandated that military and Department of Veterans Affairs hospitals screen all service personnel returning from combat. Military health professionals must evaluate them for concussion and then treat the physical, emotional, and cognitive problems that may surface. A new approach to health management and care coordination is needed that will allow medical transitions between networks of care to become more centralized and allow for optimal recovery at all severity levels. This article summarizes the care systems available for the acute management of TBI from point of injury to stateside military treatment facilities. We describe TBI assessment, treatment, and overall coordination of care, including innovative clinical initiatives now used.
\end{abstract}

Key words: cognitive assessment, community reentry, loss of consciousness, medical evacuation, polytrauma, posttraumatic amnesia, rehabilitation, TBI, TBI screening, traumatic brain injury.

\section{INTRODUCTION}

The use of unconventional weaponry has increased the risk of traumatic brain injury (TBI) for military personnel engaged in current combat operations. Although the true incidence and prevalence of combat-related TBI is

\footnotetext{
Abbreviations: $\mathrm{AE}=$ air evacuation, $\mathrm{ANAM}=$ Automated Neuropsychological Assessment Metrics, AOC = alteration of consciousness, CONUS $=$ continental United States, DOD = Department of Defense, DVBIC = Defense and Veterans Brain Injury Center, GCS = Glasgow Coma Scale, LOC = loss of consciousness, LRMC = Landstuhl Regional Medical Center, MACE $=$ Military Acute Concussion Evaluation, $\mathrm{mTBI}=$ mild TBI, MTF = military treatment facility, PTA = posttraumatic amnesia, RTD = return to duty, TBI = traumatic brain injury, VA $=$ Department of Veterans Affairs, VAMC = VA medical center.

* Address all correspondence to Philip D. Girard, Office of Telemedicine, Manchester VA Medical Center, 718 Smyth Road, Manchester, NH 03104; 603-617-0293; fax: 603-6266502. Email: Philip.girard@va.gov

DOI:10.1682/JRRD.2008.09.0114
} 
unknown, military personnel returning from deployment have reported rates of concussion between 10 and 20 percent [1]. Approximately, 4.9 percent of servicemembers are symptomatic when screened after deployment, prompting a clinical referral. Following clinical evaluation, 20,119 cases of TBI were confirmed in 2009. The Department of Defense (DOD) has recently released statistics on TBI dating back to before the Iraq and Afghanistan wars (visit www.DVBIC.org). The Defense and Veterans Brain Injury Center (DVBIC) continues to improve TBI surveillance efforts and methodology to better elucidate the impact and burden of TBI on America's warriors and veterans [2]. Providers face many unique challenges delivering and coordinating the care of servicemembers and veterans with TBI. The DVBIC is working with the military and the Department of Veterans Affairs (VA) centers to unify health systems for patients with TBI. The DVBIC is a triservice, DOD/VA congressionally mandated organization made up of a growing number of military treatment facilities (MTFs), VA Polytrauma Rehabilitation Centers, and civilian community reentry facilities. Specific information on care within the VA Polytrauma Rehabilitation Centers and outpatient clinics can be found elsewhere [3] and is beyond the scope of this article. This article summarizes the systems of care available for the acute management of TBI from point of injury to stateside MTFs. Within this context, we describe TBI assessment and treatment, and overall coordination of care for patients with TBI, including innovative clinical initiatives that have been employed during this conflict. We draw this information from multiple efforts within the DOD and VA to rapidly identify those with TBI and provide comprehensive treatment toward recovery for all those who sustain a TBI.

\section{OVERVIEW OF TRAUMATIC BRAIN INJURY}

TBI is defined as a blow, jolt, or other injury to the head that disrupts the functioning of the brain. Primary injury results when an external force is applied to the brain that is significant enough to alter neurological functioning or consciousness. Secondary brain injury may follow, exacerbating cerebral dysfunction. Secondary injury occurs when flow-metabolism mismatches arise, commonly following hypoxia or hypotension.

Much more is known about secondary brain injury occurring after severe TBI than about concussion. As described in subsequent details, TBI severity is catego- rized as mild, moderate, or severe. Mild TBI (mTBI), more commonly known as concussion, typically involves a brief change in mental status and transient symptoms. Imaging, using standard techniques, is often normal in mTBI [4]. Moderate brain injuries are associated with longer periods of unconsciousness or amnesia following the traumatic event and have a significantly higher incidence of structural injury on routine imaging. Patients with moderate TBI may experience a variety of symptoms, including mood and memory disturbances and physical and emotional problems, which may persist for months. Moderate TBI requires increased levels of clinical intervention but may be responsive to medication, psychotherapy, and compensatory strategies. A severe TBI may result in long-term problems with independent functioning and can result in moderate to severe physical or cognitive disability in some patients. Since severe TBI frequently occurs with other significant traumatic injuries, comprehensive nursing care and ongoing rehabilitation are usually required. Optimal recovery from severe TBI depends on rapid evacuation, early intervention, and ongoing specialized treatment in acute settings.

The challenges involved in managing TBI exist on many levels. The unique occurrence of physical, cognitive, and emotional symptoms associated with brain injury requires collaboration of many disciplines. Mild and moderate TBI may not be immediately obvious, because symptoms may be subtle. Thus, detection may be delayed. Together, these factors add to the difficulty of identifying mTBI to moderate TBI early.

\section{Traumatic Brain Injury Severity Indicators}

One can determine the severity of injury by comparing three indexes: the overall Glasgow Coma Scale (GCS) score, the length of time of loss of consciousness (LOC) or alteration of consciousness (AOC), and the duration of posttraumatic amnesia (PTA). Of these parameters, the GCS has been the most widely studied and validated, especially when evaluating severe TBI [5]. mTBI is characterized by a LOC of less than 30 minutes, a period of PTA that resolves within 24 hours, or a GCS score of 13 to 15. Moderate TBI is indicated by LOC of more than 30 minutes or a GCS score between 9 and 12. Severe TBI is classified by LOC longer than 24 hours, PTA greater than 7 days, or a GCS score between 3 and 8 . When severity indicators are inconsistent, the most severe characterization is used. Although sometimes difficult to assess in an austere environment, this information can 
help clinicians classify the severity of injury ranging from mild to moderate to severe. For all indices to be reported or available is uncommon. Any one of them alone can qualify assignment of severity of injury. Although the initial severity of injury is not functionally based, this broad classification of severity of injury can help determine which resources may be necessary, as well as which type of care setting is most appropriate (Table 1).

\section{Combat-Related Traumatic Brain Injury}

Persons with certain job specialities within the military have increased risk of sustaining TBI. Gunners, explosive ordinance device personnel, and special operations forces can all be at a higher risk because of the nature of their military occupation. Paratroopers report concussion at a rate twice that of nonparatroopers [6]. The causes of brain injury in combat include blasts events, falls, gunshot wounds, and motor vehicle crashes. These mechanisms affect the brain with varying levels of force. The resulting injuries can be localized or diffused. Individual physical characteristics combined with these varied mechanisms yield unique sequelae that affect multiple systems in the body. To help clinicians distinguish these effects, an initial classification is made between penetrating and closed brain injuries. Any injury that involves the penetration of a foreign object, munitions fragment, bone chip, etc. through the dura mater is called a penetrating TBI. Conversely, brain injuries caused by blunt trauma are considered closed. The pathophysiology of closed TBI differs in many ways from penetrating TBI; however, the damage to the brain can be equally severe.

Blast events can cause injury through multiple mechanisms. These mechanisms may include direct exposure (primary blast injury) to the overpressurization and negative

Table 1.

Severity of traumatic brain injury (TBI) rating scale (Department of Defense [DOD], 2008).

\begin{tabular}{lcccc}
\hline Severity & GCS $^{*}$ & LOC & AOC $^{\dagger}$ (h) & PTA \\
\hline Mild & 13 to 15 & $<30 \mathrm{~min}$ & 24 & $<24 \mathrm{~h}$ \\
Moderate & 9 to 12 & 30 min to $24 \mathrm{~h}$ & $>24$ & $24 \mathrm{~h}$ to $<7 \mathrm{~d}$ \\
Severe & 3 to 8 & $>24 \mathrm{~h}$ & $>24$ & $\geq 7 \mathrm{~d}$ \\
\hline
\end{tabular}

*Although not used in severity classification by DOD, GCS is frequently used in assessing TBI severity.

${ }^{\dagger}$ Alteration of consciousness (AOC) includes being dazed or confused or seeing stars.

GCS = Glasgow Coma Scale, LOC $=$ loss of consciousness, PTA $=$ posttraumatic amnesia. pressure waves of the explosion, which can strike the body at a velocity $>300 \mathrm{~m} / \mathrm{s}$, equal to the speed of sound in air. This impact may rapidly compress air-filled organs and/or displace the entire body. Increasing evidence demonstrates that organs surrounded by fluid, including the brain, may also be at risk for injury from primary blast [7]. Other mechanisms postulated to cause primary injury include cavitation, electromagnetic fields, noxious toxin exposure, and air emboli [8]. Secondary blast injury may then be caused by the energized (projected or falling) debris or explosive fragments that strike the head. Tertiary blast injury may also occur as the displaced body strikes the ground, a wall, or any other object. Finally, quaternary injuries may occur through the inhalation of gases or other toxic substances [9-10]. Individually, these mechanisms can result in cerebral dysfunction, producing physical, cognitive, and neuropsychiatric impairments. Together, they can cause TBI along with any number of other injuries, such as burns, abdominal wounds, or limb amputation. The effects of a blast-related injury to the brain are not fully understood and remain an area of intense research.

\section{Medical Evacuation}

Care for combat-wounded servicemembers is expedited though a medical transport system, which begins on the battlefield with initial life-saving treatment and extends through combat-support hospitals and regional medical centers into the continental United States (CONUS). Acute care continues with further medical stabilization and supportive TBI care in a rapid sequence of events that ensures injuries are quickly identified and treated. The medical assets at each level of care are different, but the goal for patients with TBI is the same: to optimize functional outcomes. Guidelines with various levels of scientific evidence exist for the management of TBI.

\section{Environments of Care}

Combat grouping, setting, and environmental factors affect the management of the care of patients with TBI within the DOD. Unique challenges involve noncombatrelated TBIs that occur in CONUS (i.e., stateside motor vehicle crashes or training accidents), those that are identified and detected during postdeployment screening, and those that come home by way of the air evacuation (AE) system because of identified injuries. The wounded warriors who come home in the AE system can be further subdivided into those who leave theater with a primary 
diagnosis of TBI or those who have polytrauma with a comorbid concussion that may not be identified until they are screened at a level-IV facility such as Landstuhl Regional Medical Center (LRMC). The Figure depicts one possible medical evacuation chain from the battlefield into the VA system, where comprehensive rehabilitation and continued medical care can occur.

\section{MILD TRAUMATIC BRAIN INJURY}

\section{DOD Traumatic Brain Injury Screening Initiatives}

TBI is diagnosed whenever an injury event leads to AOC. The diagnosis can be hampered because clinicians and patients may overlook brief periods of AOC. Symptom reporting is not required when the diagnosis is confirmed. However, concussion can result in a variety of nonspecific symptoms that commonly occur with other health prob- lems; therefore, absence of a good clinical interview can delay TBI recognition and treatment. Symptoms such as fatigue, irritability, depression, and difficulty concentrating may occur alone or in combination with the more classic signs of TBI: dizziness, nausea, headache, and other physiological problems. Individually, any of these conditions may be enough to affect reaction time and decision making, which are critical in maintaining the performance and safety of servicemembers on duty as well as preserving unit and force readiness. Recognizing the need for improved TBI detection and under the direction of Congress, the military and VA have established TBI screening and assessment systems that are intended to identify TBI in military and veteran patients at multiple points of care. TBI screening involves a clinician's quick evaluation to determine exposure to a traumatic event, AOC following the event, and the presence of current symptoms. Screening occurs in CONUS during postdeployment, during periodic

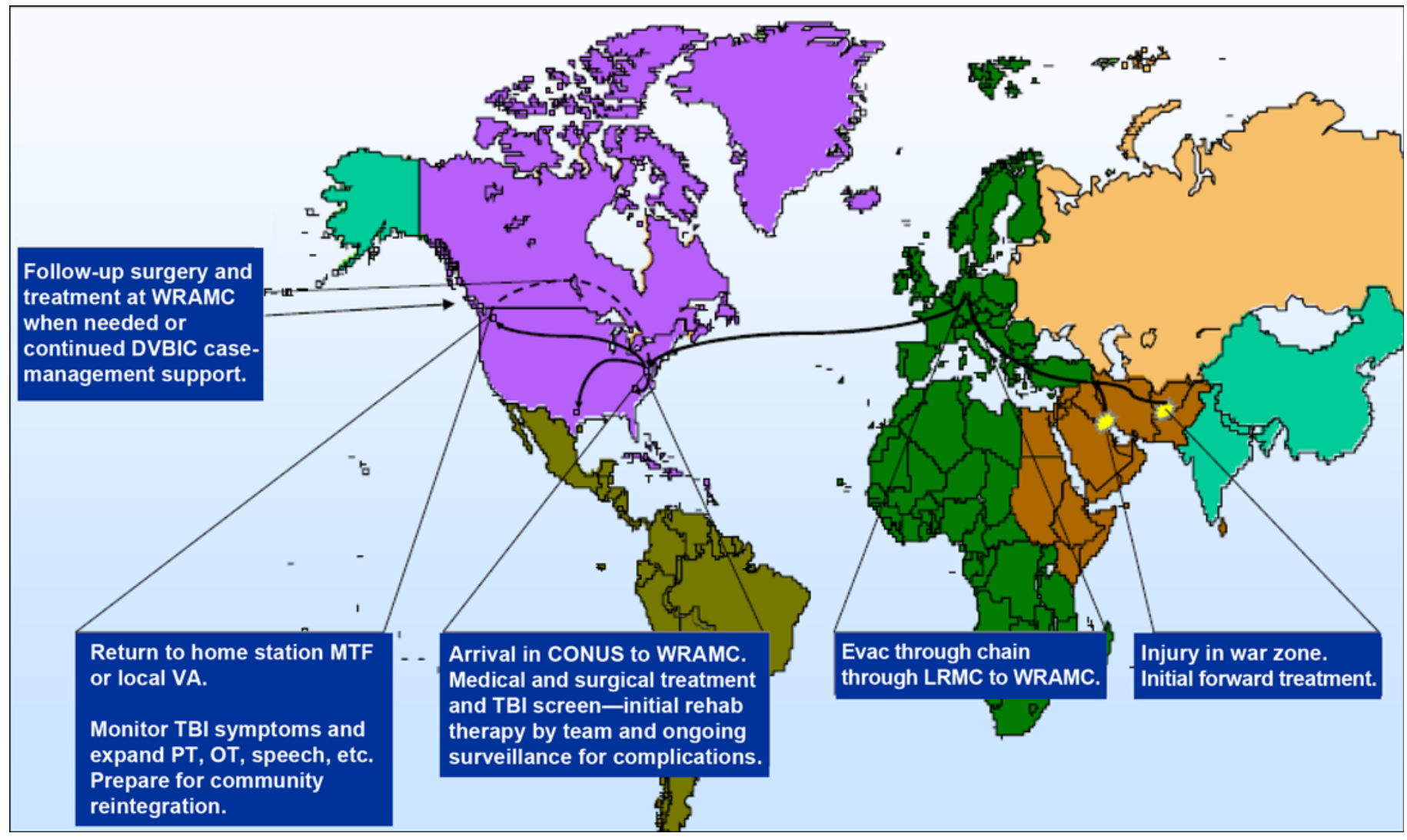

Figure.

Medical evacuation of battle-injured patients across three continents typically occurs within days from injury because of sophisticated trauma systems supported by robust training and educational initiatives. CONUS = continental United States, DVBIC = Defense and Veterans Brain Injury Center, Evac = evacuation, LRMC = Landstuhl Regional Medical Center, MTF = military treatment facility, OT = occupational therapy, PT = physical therapy, rehab $=$ rehabilitation, TBI = traumatic brain injury, VA = Department of Veterans Affairs, WRAMC = Walter Reed Army Medical Center. 
health assessments, and longitudinally, depending upon the health status and duties of individual patients. The Brief Traumatic Brain Injury Screen [11] has been recommended for routine TBI screening by the Institute of Medicine [12]. A clinical interview and examination always follow positive TBI screens for confirming or negating the diagnosis of concussion or mTBI. In the absence of temporally related symptoms, screening serves to identify those patients in whom TBI is suspected and to establish the incidence and prevalence of this injury [11]. For those with ongoing symptoms, screening can facilitate appropriate care.

\section{In-Theater Assessment}

Comprehensive assessment of TBI in an austere environment may be difficult or impossible. Since tactical situations usually prohibit comprehensive neuropsychological testing or thorough evaluations by specialists, more routine measures have been developed that allow medics and other first responders to screen for TBI immediately following a traumatic event, using the Military Acute Concussion Evaluation (MACE) developed by the DVBIC in 2006. This quick reference tool includes the Standardized Assessment of Concussion, which provides a gross measure of four cognitive domains: orientation, immediate memory, concentration, and memory recall [13]. When combined with other information, including LOC and PTA, the MACE score can provide additional information relating to cognitive performance, as well as the need for evacuation to a higher level of care. The MACE is currently undergoing further validation studies in a combat environment. Medics in theater are also equipped with guidance on moderate to severe TBI care developed by the Brain Trauma Foundation [14]. This tool, utilizing a concise algorithm, provides triage, field management, and evacuation guidance for more serious brain injuries.

\section{Treatment}

Treatment for concussion can be challenging for providers because of the paucity of evidence-based clinical strategies. Management of TBI may have several different components, which include symptom management, education, therapies, and implementation of duty restrictions. The overarching goal of the treatment plan is to facilitate maximal functional recovery and community reintegration. Assessment attempts to identify all existing physical, cognitive, and behavioral sequela so that the clinical treatment plan will appropriately target the symptom cluster. Detailed visual and vestibular evaluations are warranted in persons with suspected deficits or complaints. Neuropsychological testing may be important in the overall evaluation of TBI. Cognitive assessment becomes even more useful when baseline data are available. Specifically, the effects of concussion can be better determined through comparison of pre- and postinjury cognitive performance [15]. Identifying changes in cognitive domains (reaction time, processing speed, and memory) and emotional status (anxiety, depression, mood, anger, etc.) can help determine the full scope of the injury. Equally important, this information can help plan future care, which may not otherwise be considered when only symptom and injury information is known. Predeployment cognitive testing is now underway and is being introduced in a stepwise fashion, beginning with predeployment baseline testing. Subsequent postinjury testing and eventually periodic neurocognitive testing are also given at key time points during one's military career. Together, these comparative tests can illustrate changes in cognitive function that might not be evident when compared with a normative data bank. Comprehensive neuropsychological assessment requires careful on-site instruction and monitoring. Examples of those used in military medical centers include-

- Neurobehavioral Symptom Inventory: Used to assess the most common symptoms experienced following TBI [16].

- The State-Trait Anxiety Inventory [17] and the Automated Neuropsychological Assessment Metrics (ANAM) Mood and Sleep Scales [18]: Provide focused assessment of mood and anxiety disturbance.

- The ANAM Simple Reaction Time and Continuous Performance subtests: Used to objectively measure cognitive performance [19].

- Repeatable Battery for the Assessment of Neuropsychological Status [20-22].

Together, these tests allow clinicians to begin to identify the areas of brain function that may have been affected by the injury. Other combinations of tests have been developed and validated by the military or privately for use in sports concussion that have been introduced in theater for TBI assessment. These computer-based assessment measures differ greatly in design, function, and utility, although they each evaluate common cognitive domains that may be impaired by concussion. The DVBIC serves as the executive agent for the Office of the Secretary of Defense's directed head-to-head comparison study of the five most prominent computerized assessment tools. 
Following assessment, treatment for TBI is often provided by multiple clinicians who work as an interdisciplinary team using the most appropriate therapeutic modality. For example, posttraumatic headaches may be most effectively managed with a pharmacological regimen, whereas visual deficits may require prism glasses and ocular exercises. Some data suggest that dizziness caused by blast injury may be different and require a greater number of vestibular habituation and ocular training sessions than dizziness caused by blunt trauma [23]. Often because a complex interplay of TBI symptoms exists, targeting the symptoms in parallel provides synergistic effects. Aggressive pharmacological management of headache may significantly improve sleep, behavior, and cognition. When using pharmacotherapy, clinicians must select the appropriate medication to prevent symptom worsening, untoward side effects, and drug interactions. However, guidance in the literature as to TBI symptom-specific pharmacological treatment is sparse. Table 2 provides guidance based on the available literature and expert opinion for the evaluation and management of commonly occurring symptoms.

An educational intervention after mTBI has been shown to be highly effective in reducing symptom reporting in children and adults [24]. Educational materials should include information about concussion, such as signs and symptoms, functional strategies that may help the recovery process, the normal recovery trajectory, as well as local resources available for further questions and care. The military has given one-page educational sheets to all patients who have screened positive for TBI. This flier has been adapted at a local level so that local resources and national Web sites can be shared. The RAND report cited DVBIC as one resource able to provide appropriate education on the various aspects of concussion, as well as more severe TBI [25].

Rest and return to duty (RTD) issues are extremely important and complex within the military health system. Mission responsibilities may take precedence, and although the military medical assets are able to recommend rest and RTD, ultimate authority for activity belongs with the commanders. Balancing clinical and operational demands can greatly challenge providers as they weigh protection of a servicemember who may be vulnerable to another brain insult against mission goals and unit safety. This finding is especially true during war time when resources must be carefully evaluated and protected. The basic tenet of RTD for mTBI follows the sports concussion guidelines in which no one is returned to full duty until he or she is asymptomatic at rest and at exertion. Servicemembers are exertionally tested by running in place and performing sit-ups or push-ups for 5 minutes and then evaluated for the return of any TBI-related symptoms or decreased cognitive function on the MACE. Mental and physical rest after mTBI is an important issue as well because of the nature in the current theater of operations. Blast events, cumulative concussion, and repeat deployments are issues to consider when a servicemember is placed on duty restrictions or returned to full duty.

Rehabilitation therapies are commonly used in treatment. They include, but are not limited to, physical, occupational, speech, recreational, and other therapies. Although cognitive rehabilitation has been shown to benefit moderate and severe TBI, its role in mTBI is unclear [26].

\section{PATIENT TRACKING: LRMC AS PRIMARY CONDUIT FOR TRAUMATIC BRAIN INJURY REFERRALS FROM OPERATION IRAQI FREEDOM/OPERATION ENDURING FREEDOM}

Tracking patients with TBI from the point of identified injury onward is an essential component of care coordination. Presently, LRMC is the primary conduit through which the DVBIC identifies and tracks patients with TBI. These include patients with severe TBI who are identified in theater at the moment of injury as well as patients with mTBI who have been evacuated for other injuries or illnesses and are captured on universal TBI screening protocols once they reach LRMC.

Patient status at LRMC often informs medical decisions about transferring patients to MTFs in CONUS. In the case of severe TBI, most patients are transferred to Walter Reed Army Medical Center or National Naval Medical Center. If the patient also sustained burn-related injuries, transfers from LRMC are often directed to Brooke Army Medical Center. The relatively rapid identification of these patients with severe TBI and the transition to highly structured inpatient treatment settings help to facilitate accurate tracking of patient movements over time.

Patients with mTBI and moderate TBI may also be sent to the MTFs, although a number of concussive patients may leave LRMC bound for smaller MTFs. Once asymptomatic and cleared by the TBI team, they may return to their original duty stations and potentially be redeployed. Ongoing follow-up of these patients with mTBI is imperative for clinicians to ensure adequate resource delivery and recovery. While the literature suggests that 5 to 15 percent of patients 
Table 2.

Initial evaluation and management of concussion.

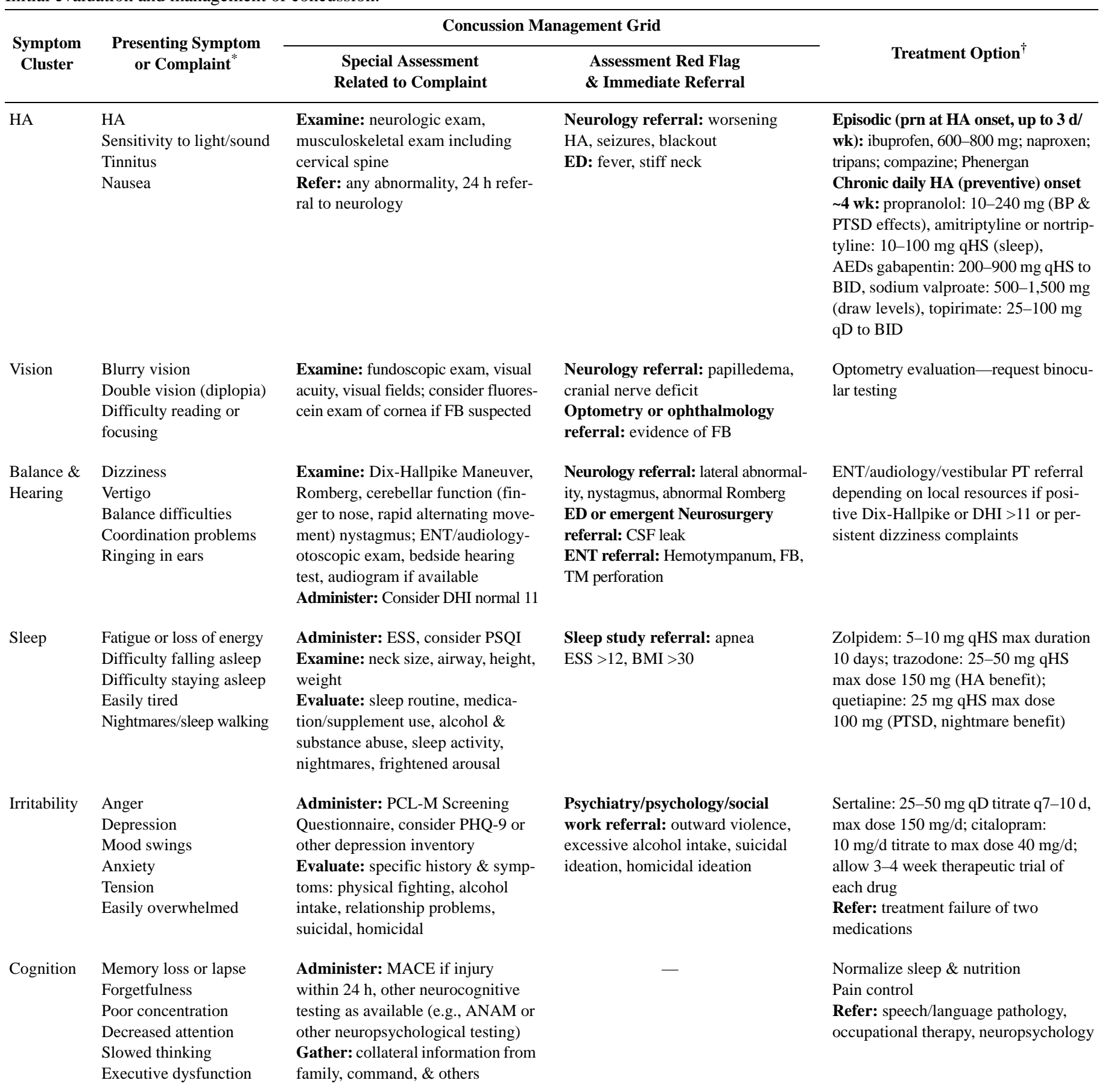

\footnotetext{
*Assess frequency, severity, aggravating factors.

${ }^{\dagger}$ Treat headache, sleep, and irritability first, because other symptoms often improve with pain control and rest. Inclusion does not imply Food and Drug Administration-approved use. See full prescribing information.

AED = antiepileptic drug; ANAM = Automated Neuropsychological Assessment Metrics; BID = bis in die (twice daily); BMI = body mass index; BP = blood pressure; $\mathrm{CSF}=$ cerebrospinal fluid; DHI = Dizziness Handicap Inventory; ED = emergency department; ENT = ear, nose, throat; ESS = Epworth Sleepiness Scale; exam = examination; FB = foreign body; HA = headache; MACE = Military Acute Concussion Evaluation; max = maximum; PCL-M = PTSD Checklist-Military Version; PHQ-9 = Patient Health Questionnaire-9; prn = pro re nata (when necessary); PSQI = Pittsburgh Sleep Quality Index; PT = physical therapy; PTSD = posttraumatic stress disorder; q7 = every seven; qD = every day; qHS = every hour of sleep (bedtime); $\mathrm{TM}$ = tympanic membrane.
} 
with concussion show persisting symptoms (beyond normal recovery times of approximately 3 months) that require therapeutic interventions [27], rates of persisting mTBI symptoms in military populations are as yet unknown, although one recent study reported rates as high as 39 percent [1]. Some patients with mTBI may appear asymptomatic in highly structured and less physically demanding environments, such as an MTF. Once they return to their duty stations and are expected to function more independently, their TBI-related deficits may become more apparent. Furthermore, a risk of reinjury exists for patients with mTBI exposed to physically and mentally demanding training maneuvers or combat if redeployed.

\section{MODERATE AND SEVERE TRAUMATIC BRAIN INJURY}

\section{Tactical Combat Casualty Care}

Tactical combat casualty care is integral in triaging and treating combat wounded with life-threatening injuries. The Guidelines for the Field Management of CombatRelated Head Trauma [14] were formulated to provide a best-evidence document to address the specific needs of assessing and managing head injury in an austere environment. The guidelines address three main areas: assessment, treatment, and triage/transport decisions. The template to create these guidelines was the Guidelines for Prehospital Management of Traumatic Brain Injury [28]. Similar content areas are discussed in both documents; however, operational concerns are incorporated into the field guidelines that are more relevant to the military community. Evidence-based guidelines for the acute care of severe TBI have also been developed [29].

The complexity of severe TBI requires an interdisciplinary approach to care. Neurology, psychiatry, neuropsychology, physical medicine and rehabilitation, and neurosurgery comprise the core medical disciplines needed for initial treatment of severe TBI. Early involvement of ancillary services that include physical therapy, occupational therapy, audiology, optometry, cognitive rehabilitation, behavioral therapy, and case management may help minimize complications, improve patient satisfaction, and maximize functional recovery. Degree of recovery from severe TBI is highly variable; however, prognostic indicators have been identified that help guide clinical practice. While the long-term effects of acute brain injury may be significant, potential for considerable improvement often exists [30], which allows patients with brain injury to lead meaningful and productive lives.

\section{Care Coordination and Traumatic Brain Injury}

The heterogeneity of TBI-related deficits uniquely challenges affected servicemembers, their families, and treatment providers. Care coordination that is specifically tailored to the unique needs of patients with TBI is essential for achieving optimal outcomes.

Patients with TBI may be treated in multiple care settings, including military, VA, and civilian healthcare systems over the course of their journey toward recovery. TBI is widely recognized, particularly moderate or severe, to compromise a patient's ability to navigate complex systems of care. Cognitive deficits, such as impaired memory, poor concentration, problems in language comprehension, and impaired judgment, hinder these individual's ability to successfully engage in treatment. People with brain injury may forget appointments, forget to take medications, be unable to drive themselves to providers' offices, or become easily confused. Mood lability and irritability can lead to outbursts at family members and treatment providers, lowered tolerance for the usual frustrations of dealing with complex care systems, and an abrupt withdrawal from treatment. Behavioral symptoms such as aggressiveness and disinhibition can elicit fear, confusion, or discomfort in others, including treatment providers who are unfamiliar with TBI. Consequently, patients with TBI are at heightened risk of premature therapy cessation or "falling through the cracks" during transitions between treatment settings and systems. Care coordination is therefore recognized as a critical need for this population [31], given the involvement of interdisciplinary providers and the likelihood of transitions between military, VA, and civilian healthcare systems for ongoing rehabilitation.

Care coordination continues to be important as patients reenter their home communities. Challenges may emerge with role identity and relationships as patients assimilate into their home environment. These challenges are complicated by the physical, cognitive, and behavioral deficits from their TBI, as well as other comorbid factors such as pain or posttraumatic stress disorder. Also, their capacity for independent living and continued participation in treatment may be altered, necessitating identification and connection to TBI resources within the community

The DVBIC has developed a network of TBI-trained care coordinators as part of a program to ensure that ongoing 
needs of patients with TBI and their families are met. Among their core responsibilities, care coordinators serve as points-of-contact to assess TBI-specific resources in communities where patients reside and facilitate access to those services, acutely and over time. They also follow patients with TBI longitudinally to help ensure positive outcomes, initiate root cause analysis when suboptimal outcomes occur, and provide connection to available services and resources within DOD, VA, and civilian healthcare systems.

\section{Patient and Family Needs}

Following the patient's medical evacuation from theater and during stabilization at a stateside MTF, the treatment team will determine the medical plan of care that best meets recovery goals. Acute management of TBI ranges from symptom management in milder cases to aggressive monitoring of intracranial pressure and brain tissue oxygenation in more severe cases. In addition to medical management, TBI care also requires that logistical issues surrounding the patient's care and patient's family needs are addressed. Depending on the severity of the TBI, the patient may or may not be able to participate in discussions and decisions regarding the treatment plan and recovery expectations. Helping family members adjust to the changes evidenced in a patient with TBI can range from learning to assist a patient with slight memory problem to caring for a bed-bound or minimally responsive individual. In many cases, if a patient is unable to return completely to his or her prior functioning level, families undergo a complex process of adapting, coping, and grieving. By providing emotional support and assisting with positive coping mechanisms, the treatment team can foster healthy reintegration of the patient and family. Recently, Congress mandated the development of family caregiver curricula to help address the need for improved educational support to include respite needs of caregivers of patients with TBI. DVBIC has been assigned as the executive agent to coordinate this effort with a White House-appointed panel. A full curricula program is expected to be launched in late 2009.

\section{Education Between Military and VA Systems of Care}

Early educational efforts focus on the immediate aspects of TBI, including current medical status, treatment plan, and expected recovery course. As transitions become imminent, family education must shift to include details of the rehabilitation process, logistics of military TBI care and, in some cases, the procedures for securing service discharge and disability. Communication with the family and patient is paramount in navigating the myriad of care and resources that are normally accessed. Patients with moderate or severe TBI often need to access acute inpatient rehabilitation at a VA Polytrauma Rehabilitation Center while still on active duty, and the patients most severely impaired will often stay within the VA healthcare system for the remainder of their time in the military and beyond. While an active duty servicemember with TBI is receiving care at a VA medical center (VAMC), coordination must occur between the VAMC, the military case manager, the DVBIC care coordinator, and the MTF treatment team. In addition, arrangements for family members to remain at the VAMC with the injured servicemember involve many logistical concerns that need to be addressed, such as Nonmedical Attendant Orders from the military, lodging and transportation arrangements, etc. Those patients with TBI who then return to an MTF or who did not need inpatient acute TBI rehabilitation are transitioned to long-term management of their TBI at local MTFs or VAMCs. Much attention has been paid to these processes for improving transitions across two large systems of care (Military Health System and Veterans Health Administration).

\section{CONCLUSIONS}

While TBI may result in physical impairment, often the more problematic consequences involve an individual's cognition, emotional functioning, and behavior. These consequences can affect all aspects of life, including the development and maintenance of interpersonal relationships and the ability to function in social settings. Community reintegration efforts are therefore aimed at maximizing individual strengths and creating supportive environments that will allow individuals with TBI to return to work and family.

Early identification and treatment are the first steps in ensuring optimal recovery. Following stabilization and medical evacuation from theater, a wide variety of clinical and rehabilitative resources is available to servicemembers. The particular resources used will depend on the severity of the injury, the clinical presentation of the patient, and the extent of recovery during the initial intervention and stabilization. Patients with TBI and their families benefit from the close partnerships formed between clinicians and other TBI health professionals who work together over distance and within multiple systems of care. The DVBIC, along with numerous other agencies, is 
working continuously to improve clinical services and strengthen the channels that support medical transitions for patients with TBI.

\section{ACKNOWLEDGMENTS}

\author{
Author Contributions: \\ Concept and design: P. D. Girard. \\ Drafting of manuscript: M. S. Jaffee, K. M. Helmick. \\ Critical revision of manuscript for important intellectual content: K. S. \\ Meyer. \\ Clinical review: K. Dinegar, K. George.
}

Financial Disclosures: The authors have declared that no competing interests exist.

Funding/Support: This material was unfunded at the time of manuscript preparation.

\section{REFERENCES}

1. Terrio H, Brenner LA, Ivins BJ, Cho JM, Helmick K, Schwab K, Scally K, Bretthauer R, Warden D. Traumatic brain injury screening: Preliminary findings in a US Army brigade combat team. J Head Trauma Rehabil. 2009;24(1): 14-23. [PMID: 19158592] DOI:10.1097/HTR.0b013e31819581d8

2. Defense and Veterans Brain Injury Center. October 2009 Armed Forces Surveillance Center data. Washington (DC): Defense and Veterans Brain Injury Center; 2009. Available at: http://www.DVBIC.org/.

3. Sigford BJ. "To care for him who shall have borne the battle and for his widow and his orphan” (Abraham Lincoln): The Department of Veterans Affairs polytrauma system of care. Arch Phys Med Rehabil. 2008;89(1):160-62.

[PMID: 18164348$]$

DOI:10.1016/j.apmr.2007.09.015

4. Haydel MJ, Preston CA, Mills TJ, Luber S, Blaudeau E, DeBlieux PM. Indications for computed tomography in patients with minor head injury. N Engl J Med. 2000; 343(2)100-105. [PMID: 10891517] DOI:10.1056/NEJM200007133430204

5. Teasdale G, Jennett B. Assessment of coma and impaired consciousness. A practical scale. Lancet. 1974;2(7872):81-84.

[PMID: 4136544]

DOI:10.1016/S0140-6736(74)91639-0

6. Ivins BJ, Schwab KA, Warden D, Harvey LT, Hoilien MA, Powell CO, Johnson CS, Salazar AM. Traumatic brain injuries in U.S. Army Paratroopers: Prevalence and character. J Trauma. 2003;55(4):617-21. [PMID: 14566111] DOI:10.1097/01.TA.0000052368.97573.D4
7. Cernak I, Wang Z, Jiang J, Bian X, Savic J. Ultrastructural and functional characteristics of blast injury-induced neurotrauma. J Trauma. 2001;50(4):695-706. [PMID: 11303167] DOI:10.1097/00005373-200104000-00017

8. Tsokos M, Paulsen F, Petri S, Madea B, Puchel K, Turk EE. Histologic, immunohistochemical, and ultrastructural findings in human blast lung injury. Am J Respir Crit Care Med. 2003;168(5):549-55. [PMID: 12842857] DOI:10.1164/rccm.200304-5280C

9. Cooper GJ, Maynard RL, Cross NL, Hill JF. Casualties from terrorist bombings. J Trauma. 1983;23(11):955-67. [PMID: 6632027] DOI:10.1097/00005373-198311000-00001

10. Mayorga MA. The pathology of blast overpressure injury. Toxicology. 1997;121(1):17-28. [PMID: 9217312] DOI:10.1016/S0300-483X(97)03652-4

11. Schwab KA, Ivins B, Cramer G, Johnson W, Sluss-Tiller M, Kiley K, Lux W, Warden D. Screening for traumatic brain injury in troops returning from deployment in Afghanistan and Iraq: Initial investigation of the usefulness of a short screening tool for traumatic brain injury. J Head Trauma Rehabil. 2007;22(6):377-89. [PMID: 18025970] DOI:10.1097/01.HTR.0000300233.98242.87

12. Institute of Medicine. Gulf War and health: Volume 7. Long-term consequences of traumatic brain injury. Washington (DC): National Academies Press; 2008.

13. McCrea M, Kelly J, Randolph C. The standardized assessment of concussion: Manual for administration, scoring and interpretation. Alexandria (VA): Brain Injury Association; 1997.

14. Knuth T, Letarte PB, Ling G, Moores LE, Rhee P, Tauber D, Trask A. Guidelines for the field management of combatrelated head trauma. New York (NY): Brain Trauma Foundation; 2005.

15. Girard PD. Military and VA telemedicine systems for patients with traumatic brain injury. J Rehabil Res Dev. 2007;44(7): 1017-26. [PMID: 18075958] DOI:10.1682/JRRD.2006.12.0174

16. Cicerone KD, Kalmar K. Persistent postconcussion syndrome: The structure of subjective complaints after mild traumatic brain injury. J Head Trauma Rehabil. 1995;10(3):1-17. DOI:10.1097/00001199-199510030-00002

17. Curran CA, Ponsford JL, Crowe S. Coping strategies and emotional outcome following traumatic brain injury: A comparison with orthopedic patients. J Head Trauma Rehabil. 2000;15(6):1256-74. [PMID: 11056407]

DOI:10.1097/00001199-200012000-00006

18. Johnson DR, Vincent AS, Johnson AE, Gilleland K, Schelgel RE. Reliability and construct validity of the Automated Neuropsychological Assessment Metrics (ANAM) mood scale. Arch Clin Neuropsychol. 2008;23(1):73-85.

[PMID: 18031982]

DOI:10.1016/j.acn.2007.10.001 
19. Reeves DL, Bleiberg J, Roebuck-Spencer T, Cernich AN, Schwab K, Ivins B, Salazar AM, Harvey SC, Brown FH Jr, Warden D. Reference values for performance on the Automated Neuropsychological Assessment Metrics V3.0 in an active duty military sample. Mil Med. 2006;171(10):982-94. [PMID: 17076451]

20. McKay C, Wertheimer JC, Fichtenberg NL, Casey JE. The repeatable battery for the assessment of neuropsychological status (RBANS): Clinical utility in a traumatic brain injury sample. Clin Neuropsychol. 2008;22(2):228-41.

[PMID: 17853143] DOI:10.1080/13854040701260370

21. McKay C, Casey JE, Wertheimer J, Fichtenberg NL. Reliability and validity of the RBANS in a traumatic brain injury sample. Arch Clin Neuropsychol. 2007;22(1):91-98. [PMID: 17141467]

DOI:10.1016/j.acn.2006.11.003

22. Randoph C, Tierney MC, Mohr E, Chase TN. The repeatable battery for the assessment of neuropsychological status (RBANS): Preliminary clinical validity. J Clin Exp Neuropsychol. 1998;20(3):310-19. [PMID: 9845158] DOI:10.1076/jcen.20.3.310.823

23. Gottshall KR, Gray NL, Drake AI, Tejidor R, Hoffer ME, McDonald EC. To investigate the influence of acute vestibular impairment following mild traumatic brain injury on subsequent ability to remain on active duty 12 months later. Mil Med. 2007;172(8):852-57. [PMID: 17803077]

24. Ponsford J, Willmott C, Rothwell A, Cameron P, Kelly AM, Nelms R, Curran C. Impact of early intervention on outcome following mild head injury in adults. J Neurol Neurosurg Psychiatry. 2002;73(3):330-32. [PMID: 12185174$]$ DOI:10.1136/jnnp.73.3.330

25. Tanielian TL, Jaycox L, editors; RAND Corporation. Invisible wounds of war. Psychological and cognitive injuries, their consequences and services to assist. Santa Monica (CA): RAND; 2008.

26. Vanderploeg RD, Schwab K, Walker WC, Fraser JA, Sigford BJ, Date ES, Scott SG, Curtiss G, Salazar AM, Warden DL; Defense and Veterans Brain Injury Center Study Group. Rehabilitation of traumatic brain injury in active duty military personnel and veterans: Defense and Veterans Brain Injury Center randomized controlled trial of two rehabilitation approaches. Arch Phys Med Rehabil. 2008; 89(12):2227-38. [PMID: 19061734]

DOI:10.1016/j.apmr.2008.06.015

27. Belanger HG, Curtiss G, Demery JA, Lebowitz BK, Vanderploeg RD. Factors moderating neuropsychological outcomes following mild traumatic brain injury: A meta-analysis. J Int Neuropsychol Soc. 2005;11(3):215-27. [PMID: 15892898] DOI:10.1017/S1355617705050277

28. Badjatia N, Carney N, Crocco TJ, Fallat ME, Hennes HM, Jagoda AS, Jernigan S, Letarte PB, Lerner EB, Moriarty TM, Pons PT, Sasser S, Scalea T, Schleien CL, Wright DW; Brain Trauma Foundation; BTF Center for Guidelines Management. Guidelines for prehospital management of traumatic brain injury, 2nd edition. Prehosp Emerg Care. 2008;12(Suppl 1):S1-52. [PMID: 18203044$]$

DOI:10.1080/10903120701732052

29. Brain Trauma Foundation; American Association of Neurological Surgeons; Congress of Neurological Surgeons; Joint Section on Neurotrauma and Critical Care, AANS/ CNS, Bratton SL, Chestnut RM, Ghajar J, McConnell Hammond FF, Harris OA, Hartl R, Manley GT, Nemecek A, Newel DW, Rosenthal G, Schouten J, Shutter L, Timmons SD, Ullman JS, Videtta W, Wilberger JE, Wright DW. Guidelines for the management of severe traumatic brain injury. J Neurotrauma. 2007;24(Suppl 1):S1-106.

30. Brown AW, Malec JW, Diehl NN, Englander J, Cifu DX. Impairment at rehabilitation and 1 year after moderate-tosevere traumatic brain injury: A prospective multi-centre analysis. Brain Inj. 2007;21(7):673-80.

[PMID: 17653941$]$

DOI:10.1080/02699050701468925

31. Iverson GL. Outcome from mild traumatic brain injury. Curr Opin Psychiatry. 2005;18(3):301-17. [PMID: 16639155] DOI:10.1097/01.yco.0000165601.29047.ae

Submitted for publication September 11, 2008. Accepted in revised form April 2, 2009. 